\title{
International stability in a digital world: emerging trends in machine intelligence, environmental sustainability and society
}

\author{
Larry Stapleton $^{1}$
}

Published online: 7 February 2018

(c) Springer-Verlag London Ltd., part of Springer Nature 2018

Whilst science can single out an elusive Higgs boson and confirm the existence dark matter in a distant galaxy, here on Earth stable, sustainable international systems which exist in symbiotic harmony with their surroundings remain far more elusive. We need a fresh understanding of the capabilities of artificial systems and their capacity to contribute to a more symbiotic relationship between and across human communities, and to build sustainable human-machine solutions in harmony with the environment.

This special issue explores ways in which advanced technologies impact and shape, potentially or actually, global systems stability with a particular emphasis upon the environment and human society. Experts from industry as well as leading scientific research centres and academic communities offer their perspectives on important, even transformative, emerging technologies and their capabilities. The special issue examines advances in intelligent automated applications aimed at addressing environmental issues and understanding online social networks. The contributions included here also reflect on the important relationships between science, technology, the environment and the economy.

These insights are drawn from the International Federation of Automation and Control (IFAC) group of Technical Committees under the auspices of the Coordinating Committee for the Social Effects of Control and Automation Systems (CC9), chaired by the editor. This issue particular focuses upon the work of the Technical Committee for Technology, Culture and International Stability (TC 9-5) which is one of the oldest communities in IFAC, originating during the cold war period of the early 1980s when problems of international stability had, as their backdrop, the security concerns

\section{Larry Stapleton}

LStapleton@WIT.ie

1 INSYTE Centre, Department of Computing and Mathematics,, Waterford Institute of Technology, Waterford, Ireland associated with nuclear proliferation. Thirty years later the scope of the committee is much wider, reflecting the growing complexity of the challenges and opportunities faced by our species. As a systems engineering community, TC 9-5 is unique in the variety and range of members' interests and includes both academics and practitioners who regularly participate in its events and publications. It belongs to group of technical committees which comprise an enormous scientific community within IFAC call which focus their attention on human factors in automation and control systems engineering. The TC 9-5 committee alone comprises over 80 members from over 20 countries in five continents and draws from a wide range of disciplines. The heartbeat of the group is a common interest in the problem of international stability, especially in relation to the proliferation of advanced technology and considerations of environmental impact, ethics and culture. It has a long standing annual conference and in 2018 the TECIS event will be held for the first time in Azerbaijan.

This starts where previous special issues from the IFAC CC9/TC 9-5 community have left off, continuing themes introduced in previous special issues of Artificial Intelligence and Society.

The opening section presents two industry perspectives, each reviewing emerging new technologies which may completely transform activities as traditional as manufacturing and commerce.

The first contribution is from Norbert Jesse, Head of Computer Science at the University of Dortmund and CEO of a high technology firm in Germany called Quinscape $\mathrm{GmBH}$. His paper examines online technology trends with particular emphasis upon "big data" and the "internet-ofthings", a natural evolution of the internet into the myriad of interconnected devices into which modern life is increasingly embedded. The proliferation of this "internetof-things" is a rapid, evolving process and the extent to which it penetrates all dimensions of our lives will be a function of the close cooperation between standardization 
organizations, open source communities and digital technology experts. His paper explores the implications of the "datafication" of commercial activities and acknowledges the new opportunities and risks that this datafication presents for society. These solutions pose significant risks and challenges. Norbert Jesse poses a central question: how do we assess technical risks when they are generated by interactions between, well, everything?

The second paper is an industry perspective from Venkat Raghavan, a materials specialist at the Pentair Global Engineering Centre in India, and Thomas Duda of Pentair Valves and Controls in Switzerland. The authors survey important production systems trends which are a result of 3D printing technologies. 3D Printing, sometimes called "Additive Manufacturing", involves the direct production of parts and products from a digitised model by building up layer upon layer until the model has become a manufactured metallic reality. This technology is transformative because it eliminates the use of traditional tooling from the manufacture of dense metal parts, replacing industrial tooling with a printer and software. Using such a process we can convert an idea to a digitised design to a manufactured artefact extremely rapidly and with immense precision. Several manufacturing industries are already utilising this technology, including sectors as diverse as aerospace, oil \& gas, marine and automotive parts. This paper offers an industrial perspective of 3D Printing Technologies and their implications, including materials, applications, advantages, disadvantages, challenges and the possible economic implications of 3D metal printing technology. It outlines the Direct Metal Laser Sintering (DMLS) Process, offering insights of some implications of 3D metal printing for less developed regions.

The third contribution follows on from the industry perspective of the preceding paper by offering a survey of the main drivers of complexity in industrial automation. Large scale manufacturing and other industrial facilities are faced with increasing levels of complexity, especially with the emergence of so-called "Industry 4.0" which involves a major leap in the diversity and amount of internet-connected devices and components, and the vast amounts of data these will generate. With all this in mind, how do managers of such facilities leverage the great potential of this digitalisation? Christoph Jansen of Siemens' Digital Factory Division and Sabina Jeschke of RWTH Aachen University survey the important drivers of this complexity in industrial automation. They synthesise important challenges for managers of facilities impacted by this major, technical transition. Their paper reveals how "Managed Industrial Security Services" are an important way to stabilize an increasingly complex industrial systems environment. This paper presents nearfuture automation scenarios as well as the major concepts involved in their protection.
Following these industry perspectives this issue presents a series of academic contributions under the heading Intelligent Systems for Environmental Sustainability. This section opens with a paper co-authored by Vassiliki Mpelogianni and Petros Groumpos of the Department of Electrical and Computer Engineering at the University of Patras in Greece. Fuzzy cognitive mapping combines fuzzy logic and cognitive mapping to represent human expert knowledge in a variety of domains. The traditional FCM methodology formally describes causal relationships that exist between concepts in the knowledge domain being modeled. Although Fuzzy Cognitive Maps have been used in many applications they remain theoretically limited, especially in the face of the increasing complexity of modern systems contexts. Their work re-assesses Fuzzy Cognitive Maps (FCMs) as a modeling methodology for complex dynamic systems. The first contribution of this paper is to refine Fuzzy Cognitive Map theory to address the limitations, including the capacity of a FCM to represent complex control system knowledge. Having addressed the theoretical limitations of FCMs, the paper then unpacks a case study to demonstrate how their revised FCM theory can be practically applied to solve environmental control problems. Their case study uses their revised FCM methodology to calculate and intelligently manage energy consumption in a building, thereby addressing high energy consumption in the building sector. In this way, the revised FCM methodology and its applications can make a significant contribution to reducing energy waste and thereby lessen the environmental impact of the built environment.

This section continues with the theme of intelligent applications for waste management with a technical paper from two Algerian academics, Mohamed Bahita and Khaled Belarbi. Their work investigates machine intelligence techniques which could potentially improve chemical waste processing, thereby reducing the impact of hazardous chemical waste upon the environment. Their study applies a neural network based on a radial basis function (RBF) to control concentrations in a type of tank reactor known as a continuous flow stirred-tank reactor (CSTR). These reactors are widely used in chemical plants throughout the world in waste water treatment processes. The stirring of slurries, gases and liquids in these reactors are typically controlled according to a mathematical model. These reactors involve highly non-linear, complex, often unstable, chemical processes. The simulation results presented in this paper suggest that these intelligent, neural-network controllers can improve process stability and ultimately reduce the impact of chemical waste on the environment.

The next contribution comes from the advanced robotics and control research laboratory at the Technical University of Sofia and is co-authored by Sevil Ahmed, Vasil Popov, Andon Topalov and Nikola Shakev. The expansion of cities 
into larger and larger conurbations and the growth of concentrated zones of industrial activity bring environmental and health hazards in the form of new kinds of pollution with implications for the quality of life of the societies inhabiting these areas. In relation to these impacts, the authors pose the following question: is it possible to transform a wireless sensor network into an intelligent, adaptive sensor system which can automatically discover and track areas where environmental parameters violate pre-determined thresholds? This paper demonstrates how a robotized wireless sensor network (WSN) can be an effective way to intelligently and automatically monitor the environmental impacts of pollution in large urban landscapes. The robot agents they investigate are components of a large distributed, integrated, control system. As a result they can improve the efficiency of existing conventional pollution prevention systems which are, in general, neither very adaptive nor intelligent. Amongst other contributions, this paper presents a potential methodology for developing robotized WSNs that can solve a variety of environmental monitoring tasks. It sets out the test results of a prototype hybrid WSN built at the Sofia control systems laboratory which incorporated robot agents capable of greater flexibility than previous monitoring systems. The test results showed that, as well as being more flexible, the distributed intelligent system was capable of gathering and transferring reliable information in a coordinated fashion.

The special issue now changes direction again, focussing upon peaceful coexistence. The issue offers three very varied contributions in this broad field, reflecting the expansive nature of the discourse in the IFAC community. It moves from conflict in social media settings and opinion formation, through a reflection on environmental instability to a final paper on data analytics as applied to critical infrastructure decisions in a post-conflict developing region.

Ways in which social media systems shape public opinion is a hot topic and the extent to which these computermediated systems influence opinion during elections (for example) has attracted great interest from scientists and policy-makers alike, as well as investigators such as the Federal Bureau of Investigation in the USA. In relation to this subject, one of the major sub-fields of artificial intelligence involves investigations of the interactions of multiple intelligent agents in a network. This is sometimes called "distributed artificial intelligence" (DAI) and involves a very large scale, intelligent, complex distributed system operating in real-time. In "Cluster Consensus in Multi-Agent Networks with Mutual Information Exchange" O. Feyza Erkan and Mehmet Akar, two scientists from Bogazici University in Turkey, use an approach which draws upon DAI studies to consider a social network system in which each agent in a network updates its opinion by relying on its neighbours' opinions, creating "cluster consensus" in a network. As the interactions between agents in the network evolve over time, their research utilises joint and integral connectivity conditions to determine the amount of clusters that are formed. In this way, their work explores "opinion dynamics" in an online system and examines the long-term behavior of online social networks as they come to form opinions. Their investigation of these dynamics suggests that the formation of different opinions, which is a natural consequence of crosssociety interactions, will reduce disagreements among the individuals within the groups and enhance the variety of opinions in the society. Their insights may provide a basis for the creation and design of a very large social network with fewer conflicts, whilst maintaining a diversity of opinion groups.

The special issue now shifts direction by considering the global impact of scientific endeavour itself. Alan Cottey is an active member of "Scientists for Global Responsibility" and a previous contributor to both IFAC TC 9-5 and this journal. His invited piece entitled "Environment change, economy change and reducing conflict at source" reflects upon an apocalyptic narrative circulating amongst many informed commentators and scientists which concerns the future of our planet. It considers the devastating impact of our species upon the living systems in which we find ourselves and the proliferation of an economic view which is fundamentally flawed. Cottey asserts that the dominant economic practice and theory, which he calls "economics-as-usual", dictates the coordinates and vocabulary for economic discourse in the public space. He argues that this language is narrow and obscure. The dramatic changes in the planet's environmental systems are kept in a separate compartment, as if economics and environment were unconnected. When this partition is removed, economics-as-usual is seen to be unsustainable. The consequence of his argument is that a radical change in economic thinking is needed. This change expands the very concept of economics itself along the lines of the ancient Greek notion of oikonomia (management, housekeeping). This paper argues that "economics-as-usual" is a major source of conflict in our world, impinging upon the systems of justice, cooperation and peace. It directs attention to the possibility of an expanded economics which enshrines a dignified life available to all people as of right. This economics re-connects 'wealth' with its etymological origins in "weal", that is: wellness and well-being. In Cottey's own words "humanity has invented and climbed aboard a fast machine without first learning how to control it". Gaining control requires a deep re-examination of individual and collective attitudes and priorities. This paper is a call to arms for scientists and engineers to rethink the cultural values of their crafts, lest we too contribute to the escalating crisis.

In less stable developing regions it is critical that information scientists develop data analytics models which focus upon the factors which can lead to improved socioeconomic conditions. In spite of a wealth of empirical data, 
many investment projects in less developed regions make incorrect assumptions about potential outcomes. As a consequence, high profile investments fail to deliver hoped-for and much needed, economic improvements. The special issue closes with a contribution from an emerging Kosovar scientist, Ylber Llimani, from the Information Systems faculty at the University of Business and Technology. His research uses various data sources to explore the relationship between transportation systems and economic development using a case study of a less developed, post-conflict region: Kosovo. In Kosovo, like many less developed regions, policy making is mostly focused on infrastructure development as a pre-cursor to economic development. The basic assumption is that investment in transportation infrastructure leads to improved economic activity and capacity which in turn creates the necessary conditions for socio-economic development. His study uses systems theory to try to understand the extent to which these assumptions are valid. He discovers that, when transportation is framed as a complex, open, dynamic system, although transportation investments can help economic development in developing regions, they are not a necessary pre-condition for development in a digitally-mediated world. In other words, countries exhibiting the properties of an open, dynamic system but which suffer from an underdeveloped transport infrastructure may not recover their economies by concentrating investment on transport infrastructure as a means of improving economic capacity and activity. However, it is even worse for countries who exhibit the properties of a closed, dynamic system. For these regions, transport investments may not be feasible due to their underlying economic instability and, as a result of their systemic properties, they may experience even more difficulties growing the economy. To unpack this he uses the country case study to show how its closed dynamic system properties impact the relationship between transport investment and economic development. From a systems perspective, closed, dynamic system countries lack equity, i.e., lack equal distribution, in their investment across the provinces in the region and lack information transparency in investment decisions and outcomes. As a result, although Kosovo enjoys relatively good (and improving) transportation infrastructure links with its neighbours, as a closed dynamic system this is unlikely to deliver the hoped-for returns on investment in terms of economic development. His research further concludes that transport system planning in countries identified as having a closed system transport planning policy need to consider a range of other factors including land use, urban modeling, environmental issues and others, if they are to experience improved economic conditions and quality of life for the citizens and thereby deliver regional stability following conflict. He offers a new analytic system framework for understanding these dynamics.

\section{Closing remarks and acknowledgements}

The subjects covered in this special issue are merely indicative of the range of interests of my colleagues in IFAC and the technical committees which are organized under the CC9 umbrella. The special issue is by no means representative of the full range of activities of technical committees under CC9.

The technical committee which contributed to this issue, TC 9-5, currently chaired by Professor Peter Kopacek of the Technical University of Vienna (TU Wien), comprises over 80 members from over 20 countries. I have not included important developments in end-of-life management and recycling, cost-oriented automation, mechatronics management and education, micro-finance, telemedicine and virtual hospitals, robotics, service-engineering or energy-systems. Neither does this special issue touch on geographical information systems, technology driven transformation in less developed regions, global forecasting or important theoretical work in mathematics which are all subjects covered by TC 9-5. However, I trust that the selection that is included demonstrates the breadth and depth of research on technology, culture and international stability currently underway in the field.

A sincere thank you goes to the reviewers for their speedy turnaround of the papers, to the senior editorial team at AI and Society for their guidance and support and, especially, to the authors for their inspiring work.

I am also deeply grateful for the support of the technical committee itself, and especially the chairperson and vicechairperson who work so hard to promote the work: Professor Peter Kopacek (Head of Institute of Handling Devices and Robotic at the Technical University of Vienna) and Dr. Marion Hersh (Head of the TC 9-5 Working Group on Ethics, University of Glasgow). 